\title{
PERIODIC SOLUTIONS OF VOLTERRA INTEGRAL EQUATIONS
}

\author{
M.N. ISLAM \\ Department of Mathematics \\ University of Dayton \\ Dayton, OH 45469 \\ (Received December 2, 1986 and in revised form Apri1 4, 1987)
}

ABSTRACT. Consider the system of equations

$$
x(t)=f(t)+\int_{-\infty}^{t} k(t, s) x(s) d s,
$$

and

$$
x(t)=f(t)+\int_{-\infty}^{t} k(t, s) g(s, x(s)) d s .
$$

Existence of continuous periodic solutions of (1) is shown using the resolvent function of the kernel k. Some important properties of the resolvent function including its uniqueness are obtained in the process. In obtaining periodic solutions of (1) it is necessary that the resolvent of $k$ is integrable in some sense. For a scalar convolution kernel $k$ some explicit conditions are derived to determine whether or not the resolvent of $k$ is integrable. Finally, the existence and uniqueness of continuous periodic solutions of (1) and (2) are obtained using the contraction mapping principle as the basic tool.

KEYWORDS AND PHRASES. Volterra integral equation, periodic solution, resolvent, integrability of resolvent, limit equation.

1980 AMS SUBJECT CLASSIFICATION CODES. 45D05, 34Al 0.

1. INTRODUCTION.

In this paper we study the existence and uniqueness of periodic solutions of the integral equations

$$
x(t)=f(t)+\int_{-\infty}^{t} k(t, s) x(s) d s, \quad-\infty<t<\infty
$$

and

$$
x(t)=f(t)+\int_{-\infty}^{t} k(t, s) g(s, x(s)) d s, \quad-\infty<t<\infty,
$$


where $x, g$ and $f$ are vectors in $R^{n}, k$ is an $n$ by matrix function with elements in $R$, and $R^{n}$ is the vector space of $n$-dimensional column vectors. We list our basic assumptions in section 2. The results and their proofs are presented in Sections 3 , 4 and 5 .

In Section 3 we present two basic results, Theorems 1 and 2, which are used in Theorem 3 of Section 4 to obtain the existence of a continuous periodic solution of (1.1). Theorem 1 deals with the resolvent kernel associated with the Volterra equation

$$
y(t)=f(t)+\int_{0}^{t} k(t, s) y(s) d s, \quad t \varepsilon R^{+}=[0, \infty) .
$$

In Theorem 2 we obtain (1.1) as a limit equation of (1.3). The resolvent equation corresponding to $(1.3)$ is

$$
r(t, s)=-k(t, s)+\int_{s}^{t} k(t, u) r(u, s) d u, \quad 0<s<t,
$$

and its solution $r(t, s)$ is called the resolvent kernel. The importance of the resolvent derives from the fact that the solution $y(t)$ of (1.3) is given by

$$
y(t)=f(t)-\int_{0}^{t} r(t, s) f(s) d s, \quad t>0
$$

The existence of continuous $r(t, s)$ as a solution of (1.4) is a known result (see [1, Chapter IV, Theorem 3.1]). In Theorem 1 we prove the uniqueness of $r(t, s)$ which is used to establish an important property, (4.4), of $r(t, s)$. We use (4.4) together with (1.6) and other properties derived in Lemmas 2 and 3 in obtaining periodic solutions of (1.1). Notice that all the properties of the resolvent function derived in this paper including the integrability properties obtained in Theorem 4 are significant results by themselves.

We assume that $r(t, 8)$ is integrable in the sense that

$$
\sup _{t>0} \int_{0}^{t}|r(t, s)| d s<\gamma<\infty .
$$

Some results regarding the property (1.6) are available in $[2,3]$. In case $k(t, 8)=a(t-$ s) is of convolution type for which the resolvent $r(t, s)=b(t-s)$ is also of convolution type, it can be verified that if both $a(t)$ and $b(t)$ are of class $L^{1}\left(R^{+}\right)$then all the results of Section 4 hold (Remark 2). A necessary and sufficient condition for $b(t)$ to be of class $L^{1}\left(R^{+}\right)$was obtained by Paley and Wiener [4] in the following result:

THEOREM 0. Suppose $a(t)$ is in the class $L^{1}\left(R^{+}\right)$. Then the resolvent $b(t)$ is in the class $L^{1}\left(R^{+}\right)$if and on $1 y$ if the determinant

$$
\operatorname{det}\left(I-\int_{0}^{\infty} e^{-z t} a(t) d t\right) \neq 0
$$

for all complex number $z$ satisfying $\operatorname{Re} z>0$. 
The integrability of $b(t)$ (i.e. $b(t)$ is in the class $L^{1}\left(R^{+}\right)$) is also studied in [5-9]. Analyzing the transcendental relation (1.7) we derive in Theorem 4 a few explicit conditions regarding the integrability of $b(t)$.

In Section 5 we use the familiar contraction mapping principle to show the existence and uniqueness of periodic solutions of (1.1) and (1.2). We obtain these results in Theorems 5 and 6 .

Some results related but different from the results of the present paper on periodic solutions are available in [10-17].

2. UNDERLYING ASSUMPTIONS.

For a vector $x$ in $R^{n}$, let $|x|$ denote a norm of $x$ equivalent to the Euclidean norm. For any $n$ by $n$ matrix $J$, let $|J|=\{|J x|:|x|<1\}$ be the matrix norm which corresponds to the vector norm $|x|$.

Throughout this paper we make the following assumptions of $f, k$, and $g$ :

(Al) $f(t)$ is continuous and $T$-periodic on $R$ for some $T>0$;

(A2) $k(t, s)$ is continuous in $(t, s)$ for $-\infty<s<t<\infty, k(t, s)=0$ for $s>t$;

(A3) $k(t+T, s+T)=k(t, s)$ for $-\infty<s<t<\infty$;

(A4) there exists a constant $B>0$ such that

$$
\sup _{t>0} \int_{0}^{t}|k(t, s)| d s<B
$$

(A5) for each $\varepsilon>0$ there exists a $\delta>0$ such that whenever $|h|<\delta$ then

$$
\int_{0}^{t}|k(t+h, s)-k(t, s)| d s+\int_{t}^{t+h}|k(t+h, s)| d s<\varepsilon
$$

for all $t>0$; (Note that the second integral becomes zero for $h<0$ since $k(t, s)=0$ for s>t).

(A6) $g(t, x)$ is defined on $R \times R^{n}$, for each $x$ in $R^{n}$ the function $g(t, x)$ is $T-$ periodic in $t$, and $g(t, 0)=0$ for all $-\infty<t<\infty$;

(A7) for each $\alpha>0$, there exists an $n>0$ such that

$$
|g(t, x)-g(t, y)|<\alpha|x-y|
$$

uniformly for $-\infty<t<\infty$ whenever $|x|,|y|<n$.

It may seem that (A2)-(A4) possibly imply (A5). To see that (A5) is independent of $(\mathrm{A} 2)-(\mathrm{A} 4)$, consider the following example suggested by C.E. Langenhop: Let $\phi(s)=0$ if $|\mathrm{s}|>T / 2, \phi(s)>0$ if $|s|<T / 2$ and 1 et $\phi(s)$ be continuous on $R$. For $-\infty<s<\infty$ and $0<t<T$ define

$$
k(t, s)=(1+t / T) \phi(s-t+T)+\phi\left(s-T^{2} /(t-T)\right) .
$$


Now, extend the definition of $k$ using the relation $k(t \pm T, s \pm T)=k(t, s)$. Note that $k(t, s)>0$. It can be shown that (1) $k(t, s)$ is continuous for $-\infty<s<t<\infty$ with $k(T, s)=2 \phi(s)$, (ii) there exists a constant $\beta>0$ such that $\int_{0}^{t} k(t, s) d s<\beta$ for all $t \geq 0$, and (iii) $\int_{0}^{t} k(t, s) d s$ is not uniformly continuous on $R^{+}$. The definition of $k(t, s)$ along with (i) and (ii) show that $k(t, s)$ satisfies $(A 2)-(A 4)$. However, $k(t, s)$ $\frac{\text { does }}{R^{+}}$not satisfy (A5) since (A5) would imply $\int_{0}^{t} k(t, s) d s$ is uniformly continuous on

\section{TWO BASIC RESULTS.}

Although the existence of a continuous solution $r(t, 8)$ of $(1.4)$ is a known result the uniqueness of such $r(t, s)$ does not seem to be explicitly shown anywhere. In Theorem 1 we establish the uniqueness of $r(t, s)$.

THEOREM 1. If $k(t, s)$ is continuous on $0<s<t<\infty$, then there exists a unique continuous solution $r(t, s)$ of $(1.4)$ on $0<s<t<\infty$.

PROOF. We only prove the uniqueness of $r(t, s)$. By way of contradiction, suppose there are two solutions $r(t, s)$ and $w(t, s)$ of (1.4) with $r(t, s) \neq w(t, s)$ for all $0<s<t<\infty$.

Then for any continuous $q$ we have

$$
y(t)=q(t)-\int_{0}^{t} r(t, s) q(s) d s, \quad t \geqslant 0,
$$

and

$$
y(t)=q(t)-\int_{0}^{t} w(t, s) q(s) d s, \quad t>0 .
$$

as the unique solution of the Volterra integral equation

$$
y(t)=q(t)+\int_{0}^{t} k(t, s) y(s) d s, t>0 \text {. }
$$

The uniqueness of the solution $y(t)$ is a well known result (see e.g., [18, Theorem $2.1 .11)$. Thus we have

$$
\int_{0}^{t} U(t, s) q(s) d s=0,
$$

where $U(t, s)=r(t, s)-w(t, s)$. Since $\quad r(t, s) \neq w(t, s)$ for all $0<8<t<\infty$, there is a $\left(t_{1}, s_{1}\right)$ with $0<s_{1}<t_{1}$ and an element $u_{\ell_{m}}$ such that $u_{\ell m}\left(t_{1}, s_{1}\right)=\alpha \neq 0$ where $\left(u_{i j}\right)=U ; 1<i, j<n$. Clearly, we may assume $\alpha>0$. Substituting $t_{1}$ for $t$ in the eth row of (3.1) we obtain 


$$
\int_{0}^{t_{1}}\left(u_{\ell 1}\left(t_{1}, s\right) q_{1}(s)+\ldots+u_{\ell m}\left(t_{1}, s\right) q_{m}(s)+\ldots+u_{\ell n}\left(t_{1}, s\right) q_{n}(s)\right) d s=0 .
$$

Since $u_{\ell_{m}}(t, s)$ is continuous in $(t, s)$ and $u_{\ell_{m}}\left(t_{1}, s_{1}\right)=\alpha>0$, there exists an $\varepsilon>0$ with $\varepsilon<s_{1}$ such that $u_{\ell_{m}}\left(t_{1}, s\right)>0$ for $0<s_{1}-\varepsilon<s<s_{1}+\varepsilon$. Let us choose a continuous function $q$ such that $q_{m}\left(s_{1}\right)=1, q_{m}(s) \geqslant 0, q_{m}(s)=0$ for $0<s<s_{1}-\varepsilon, s>s_{1}+\varepsilon$, and $q_{j}(s) \equiv 0$ for $j=1,2, \ldots, n, j \neq m$. Then it follows from the choice of $q$ and from the property of $u_{\ell_{m}}$ that the left side of (3.2) is nonzero, which is a contradiction.

LEMMA 1. Suppose $k(t, s)$ satisfies (A2) and (A3). Then (A4) holds if and only if

$$
\sup _{-\infty<t<\infty} \int_{-\infty}^{t}|k(t, s)| d s<B
$$

holds.

PROOF. Trivially, (3.3) Implies (A4). To see that (A4) Implies (3.3), consider an arbitrary $t$ in $R$. Then choose a positive integer $n_{0} s u c h$ that $t+n T>0$ for all $n \geqslant n_{0}$. It follows from (A3) that

$$
\int_{-n T}^{t}|k(t, s)| d s=\int_{0}^{t+n T}|k(t+n T, s)| d s<B
$$

for all $n>n_{0}$. This implies (3.3).

By virtue of Lemma 1 the integrals involved in Theorem 2 and in subsequent results of this paper are defined and finite.

THEOREM 2. Suppose (Al)-(A5) hold. If $y(t)$ is the continuous bounded solution of (1.3) on $\mathrm{R}^{+}$, then there exists a sequence of integers $n_{j}+\infty$ as $j+\infty$ such that $y\left(t+n_{j} T\right)+x(t)$, a continuous solution of $(1.1)$ on $R$, as $j \rightarrow \infty$, and the convergence 1 s uniform on compact subsets of $R$.

PROOF. Since $y(t)$ is a continuous and bounded function on $R^{+}$, it follows from (A4) and (A5) that $\int_{0}^{t} k(t, s) y(s) d s$ is bounded and uniformly continuous on $R^{+}$. Thus, from (1.3) and (Al) we see that the function $y(t)$ is bounded and uniformly continuous on $R^{+}$. Hence, for any $\alpha>0$ the sequence $\{y(t+n T), n T>\alpha, n \varepsilon N\}$ of translated functions is equicontinuous and uniformly bounded on $-\alpha<t<\infty$, where $N$ denotes the set of positive integers. Therefore, by Ascol1's theorem there exists a sequence of integers $n_{j}$ and a continuous function $x(t)$ such that

$$
\max \left|y\left(t+n_{j} T\right)-x(t)\right|<\frac{1}{j}
$$

This proves that $y\left(t+n_{j} T\right) \rightarrow x(t)$ as $j+\infty$, and the convergence is uniform in $t$ on each compact subset of $R$.

Let $L$ be a bound for $|x(s)|$ when $-\infty<s<\infty$. For any $-\infty<t<\infty$, if $t+n_{j} T>0$ with $\left.j\right\rangle|t|$ then a few calculations yield

$$
\left|\int_{-\infty}^{t} k(t, s) x(s) d s-\int_{-n_{j} T}^{t} k(t, s) y\left(s+n_{j} T\right) d s\right|
$$




$$
<2 L \int_{-\infty}^{-j}|k(t, s)| d s+\frac{1}{j} \int_{j}^{t}|k(t, s)| d s
$$

This last expression tends to zero as $j^{+\infty}$. Therefore, taking the limit in the sequence of translated equations (obtained from (1.3))

$$
y\left(t+n_{j} T\right)=f(t)+\int_{-n_{j} T}^{t} k(t, s) y\left(s+n_{j} T\right) d s
$$

as $j^{+\infty}$, we get $(1.1)$ as required to show that $x(t)$ satisfies (1.1) on $R$.

4. PERIODIC SOLUTIONS USING THE RESOLVENT KERNEL.

LEMMA 2. If $k(t, s)$ satisfies (A2) and (A3) then $r(t, s)$ satisfies the following properties:

$$
r(t, s) \text { is continuous for } 0<s<t<\infty, r(t, s)=0 \text { for } s>t \text {, }
$$

and

$$
r(t+T, s+T)=r(t, s) \text { for } 0<s<t<\infty \text {. }
$$

PROOF. It follows from Theorem 1 that (A2) implies (4.1). Substituting $t+T$ for $t$ and $s+T$ for $s$ in $(1.4)$, and then using (A3) we obtain

$$
v(t, s)=-k(t, s)+\int_{s}^{t} k(t, u) v(u, s) d u,
$$

where $v(t, s)=r(t+T, s+T)$. So, $v(t, s)$ satisfies (1.4) for $0<s<t<\infty$. Now, the property (4.2) follows from the uniqueness property of Theorem 1.

LEMMA 3. Suppose $k(t, s)$ satisfies $(A 2)-(A 5)$. Suppose also $r(t, s)$ satisfies (1.6). Then for each $\varepsilon>0$ there exists a $\delta>0$ such that

$$
\int_{0}^{t}|r(t+h, s)-r(t, s)| d s+\int_{t}^{t+h}|r(t+h, s)| d s<\varepsilon
$$

for all $t>0$ whenever $|\mathrm{h}|<\delta$.

The proof of Lemma 3 involves the use of (1.4) and the application of Fubini's theorem. We omit its proof because a parallel result is available in [2, Theorem 2].

In Lemma 2 we proved that $r$ satisfies the relation $r(t+T, s+T)=r(t, s)$ for $0<s<t<\infty$. Let us extend this $r$ using the relation $r(t, s)=r(t+n T, s+n T)$ for $-\infty<s<t<0$ where $n$ is a positive integer and large enough so that $t+n T>s+n T>0$. This extended $r$ is now defined and continuous for $-\infty<s<t<\infty$. Also, $r(t, s)$ satisfies the relation

$$
r(t+T, s+T)=r(t, s) \text { for }-\infty<s<t<\infty \text {. }
$$


It now follows from Lemma 1 that $(1.6)$ holds if and only if

$$
\sup _{-\infty<t<\infty} \int_{-\infty}^{t}|r(t, s)| d s<r
$$

holds. Thus, the integrals involved in Theorem 3 make sense.

THEOREM 3. Suppose (A1)-(A5) hold. Suppose also $r(t, s)$ satisfies (1.6). Then (1.1) has a continuous periodic solution $x(t)$ on $R$. (We use the term "Periodic solution" to refer to T-periodic solution).

PROOF. It follows from (1.5), (1.6) and (Al) that the solution $y(t)$ of (1.3) is bounded on $R^{+}$. Again, (Al) and Lemma 3 imply that $\int_{0}^{t} r(t, s) f(s) d s$ is uniformly continuous on $R^{+}$. So, by Theorem 2 there exists a sequence of integers $n_{j}$ such that $y\left(t+n_{j} T\right)+x(t)$, a continuous solution of $(1.1)$ on $R$, as $j \rightarrow \infty$.

Let $M$ be a bound for $|f(s)|$ when $-\infty<s<\infty$. For $-\infty\left\langle t<\infty\right.$, if $t+n_{j} T>0$ with $\left.j\right\rangle|t|$ then

$$
\left|\int_{-\infty}^{t} r(t, s) f(s) d s-\int_{n j}^{t} T^{T} r(t, s) f(s) d s\right|<2 M \int_{-\infty}^{-j}|r(t, s)| d s,
$$

which tends to zero as $j+\infty$. Taking the limit in the sequence of translated equations (obtained from (1.5))

$$
y\left(t+n_{j} T\right)=f(t)-\int_{-n_{j} T}^{t} r(t, s) f(s) d s
$$

as $j+\infty$, we obtain

$$
x(t)=f(t)-\int_{-\infty}^{t} r(t, s) f(s) d s
$$

If follows from ( $\mathrm{Al}$ ) and (4.4) that $x(t)$ in (4.5) is T-periodic.

REMARK 1. In the proof of Theorem 3 one may notice that it is only the continuity instead of uniform continuity of $\int_{0}^{t} r(t, s) f(s) d s$ that is needed. This continuity could be obtained from the condition

$$
\begin{gathered}
\lim _{h \rightarrow 0}\left[\int_{0}^{t}|r(t+h, s)-r(t, s)| d s+\int_{t}^{t+h}|r(t+h, s)| d s\right]=0 \\
\text { for each } t>0,
\end{gathered}
$$

which is relatively weaker version of condition (4.3). Note that for condition (4.6) to hold, assumption (A5) could be replaced by the following property:

$$
\begin{gathered}
\lim _{h \rightarrow 0}\left[\int_{0}^{t}|k(t+h, s)-k(t, s)| d s+\int_{t}^{t+h}|k(t+h, s)| d s\right]=0 \\
\text { for each } t>0
\end{gathered}
$$

However, to prove Theorem 2 which is used in Theorem 3 we need (A5) so that $\int_{0}^{t} k(t, s) y(s) d s$ can be uniformly continuous. The uniform continuity of 
$\int_{0}^{t} k(t, s) y(s) d s$ is needed for the equicontinuity of $\{y(t+n T), n T>\alpha, n \varepsilon N\}$ on $-\alpha<t<\infty$

REMARK 2. If $k(t, s)=a(t-s)$ with $a(t)$ in the class $L^{l}\left(R^{+}\right)$then $a(t)$ satisfies (A3) and (A5). Similarly, if the resolvent $b(t)$ of $a(t)$ is of class $L^{1}\left(R^{+}\right)$then $b(t)$ satisfies (4.3) and (4.4). Therefore, the results of Theorems 2 and 3 include convolution equations as special cases.

The following are a few conditions derived from Theorem 0 to determine whether or not $b(t)$ is of class $L^{1}\left(R^{+}\right)$.

THEOREM 4. Suppose $a(t)$ is a real valued continuous function on $R^{+}$with $a(t)$ in the class $L^{1}\left(R^{+}\right)$. Let $b(t)$ be the resolvent of $a(t)$.

(i) If $\int_{0}^{\infty}|a(t)| d t>1$, then $b(t)$ is not in the $\operatorname{class~} L^{1}\left(R^{+}\right)$.

(11) If $\int_{0}^{\infty}|a(t)| d t<1$, then $b(t)$ is in the class $L^{1}\left(R^{+}\right)$.

(i1i) Suppose $a(t)$ does not change its sign on $R^{+}$. If $-1<\int_{0}^{\infty} a(t) d t<1$, then $b(t)$ is in the class $L^{1}\left(R^{+}\right)$.

PROOF. Since $a(t)$ is a scalar function, the condition (1.7) becomes $1-a^{\star}(z) \neq 0$ for $\operatorname{Re} z>0$, where

$$
a^{*}(z)=\int_{0}^{\infty} e^{-z t} a(t) d t
$$

let $q(z)=1-a^{*}(z)$.

PROOF of (i). It is sufficient to prove that there exists at least one root of $q(z)$ in the closed right half plane. If $\int_{0}^{\infty} a(t) d t=1$ then $q(0)=1-a^{*}(0)=0$. So, $z=0$ is a root of $q(z)$. If $\int_{0}^{\infty} a(t) d t>1$ then $q(0)<0$. Considering $y=0, x>0$ where $x+1 y=z$, we obtain $a^{*}(z)=a^{*}(x)$ which tends to zero as $x+\infty$. Thus, $q(x)=1-a^{*}(x)$ +1 as $x+\infty$. Since $q(x)$ is a real valued continuous function on $R^{+}, q(0)<0$, and $q(x) \rightarrow 1$ as $x \rightarrow \infty$, it follows that there is a real positive root of $q(x)$ on $R^{+}$. PROOF of (1i). From the hypothesis we get $\left|a^{\star}(z)\right|\langle 1$ for $\operatorname{Re} z>0$. So, $|q(z)|=\left|1-a^{*}(z)\right|>1-\left|a^{*}(z)\right|>0$ for $\operatorname{Re} z>0$. Therefore, $q(z)$ has no root $z$, $\operatorname{Re} z>0$.

PROOF of (iii). We assume that $a(t) \neq 0$. Otherwise $b(t) \equiv 0$. Since a(t) does not change its sign, the condition $-1<\int_{0}^{\infty} a(t) d t<1$ is the same as the one in (1i). So, we consider only the case $\int_{0}^{\infty} a(t) d t=-1$.

Let $\phi(t)=-a(t)$. Clearly, $\phi(t)>0$ for all $t>0$ and $\int_{0}^{\infty} \phi(t) d t=1$. Now

$$
\begin{aligned}
& q(z)=1-a^{*}(z)=1+\phi^{*}(z) \\
& =1+\int_{0}^{\infty} e^{-x t} \cos y t \phi(t) d t+1 \int_{0}^{\infty} e^{-x t} \sin y t \phi(t) d t .
\end{aligned}
$$


Thus, to get $q(z)=0$ one must have

$$
1+\int_{0}^{\infty} e^{-x t} \cos y t \phi(t) d t=0
$$

First we show that for $y \neq 0, x>0$

$$
\left|\int_{0}^{\infty} e^{-x t} \cos y t \phi(t) d t\right|<1
$$

which contradicts (4.8). The case $y=0, x>0$ is considered later.

For $y \neq 0$ consider the set

$$
E=\left\{t \in R^{+}: t=n \pi / y, n=\ldots .-2,-1,0,1,2, \ldots .\right\}
$$

Clearly, $E$ is a countable subset of $R^{+}$and $|\cos y t|=1$ for $t \varepsilon E$. It is easy to see that there exists a positive $t_{1} \notin E$, such that $\phi\left(t_{1}\right) \neq 0$. Otherwise $\phi(t)=0$ for all $t \varepsilon E^{C}$, the complement of $E$ with respect to $R^{+}$. This would imply $\phi(t) \equiv 0$ on $R^{+}$, a contradiction to $\int_{0}^{\infty} \phi(t) d t=1$. Thus, there exists a $t_{1}>0$ such that $\phi\left(t_{1}\right)>0$ and $\left|\cos y t_{1}\right|<1$. Let us choose a $\delta>0$ such that $t_{1}-\delta>0$ and $|\cos y t|<1$ for $\left|t-t_{1}\right|<\delta$. Suppose $\gamma=\max |\cos y t|$ for $\left|t-t_{1}\right|<\delta$. Note that $\gamma$ exists and $\gamma<1$. Also,

$$
\int_{t_{1}-\delta}^{t_{1}+\delta} \phi(t) d t=\mu>0 .
$$

Since $\gamma<1$ and $\mu>0$ then $\gamma \mu<\mu$. Hence,

$$
\begin{aligned}
\int_{0}^{\infty}|\cos y t| \phi(t) d t & <\int_{0}^{t_{1}-\delta} \phi(t) d t+\int_{t_{1}-\delta}^{t_{1}+\delta} \gamma \phi(t) d t+\int_{t_{1}+\delta}^{\infty} \phi(t) d t \\
& <\int_{0}^{\infty} \phi(t) d t=1 .
\end{aligned}
$$

This shows that the condition (4.9) holds.

For $y=0, x>0$ the function $q(z)=q(x)>1$. From (1) we know that $\int_{0}^{\infty} e^{-x t} \phi(t) d t$ tends to zero as $x \rightarrow \infty$. This shows that $q(x)+1$ as $x \rightarrow \infty$. So, $q(z)$ has no root for $y=0, x>0$.

5. PERIODIC SOLUTIONS USING THE CONTRACTION MAPPING PRINCIPLE.

Let $X=\left\{x(t): R \rightarrow R^{n}, x(t)\right.$ is continuous and bounded on $\left.R\right\}$. For $x$ in $x$ let $\|x\|=\sup \{|x(t)|:-\infty<t<\infty\}$. Then $(X,\|\cdot\|)$ is a Banach space. For simplicity we write $X$ instead of $(X,\|\cdot\|)$. Let $P_{T}=\{x$ in $X: x(t+T)=x(t)$ for all $-\infty<t<\infty\}$. 
Then $\mathrm{P}_{\mathrm{T}}$ is also a Banach space.

LEMMA 4. Suppose (Al) - (A4) hold. If $x(t)$ is a continuous bounded solution of (1.1) on $R$ then $x(t+T)$ is also a continuous bounded solution of (1.1) on $R$.

Similarly, if (Al) - (A4), and (A6) hold then $x(t+T)$ is a continuous bounded solution of (1.2) on $R$ whenever $x(t)$ is a continuous bounded solution of (1.2) on $R$.

The proof of Lemma 4 is easy and is left for the readers to verify.

LEMMA 5. If $k(t, s)$ satisfies (A2) - (A5) then for each $\varepsilon>0$ there exists a $\delta>0$ such that

$$
\int_{-\infty}^{t}|k(t+h, s)-k(t, s)| d s+\int_{t}^{t+h}|k(t+h, s)| d s<\varepsilon
$$

for all $-\infty<t<\infty$ whenever $|h|<\delta$.

The arguments of the proof of Lemma 1 carry over to the proof of Lemma 5 .

THEOREM 5. If (A1) - (A5) hold an if $B$ of (A4) is less than 1 then there exists a unique continuous periodic solution $x(t)$ of $(1.1)$ on $R$. Moreover, $x(t)$ is the on $1 y$ continuous bounded solution on $R$.

PROOF. Since $B<1$, it follows from Lemma 1 that

$$
\sup _{-\infty<t<\infty} \int_{-\infty}^{t}|k(t, s)| d s<B<1 .
$$

For any $\phi$ in $X$ define a map $A$ on $X$ by

$$
A \phi(t)=f(t)+\int_{-\infty}^{t} k(t, s) \phi(s) d s,
$$

where $X$ is the Banach space introduced at the beginning of this section. Since $\phi(s)$ is a continuous and bounded function on $R$, it follows from Lemma 5 that $\int_{-\infty}^{t} k(t, s) \phi(s) d s$ is continuous (in fact uniformly continuous) and bounded on $R$. Thus, the function $A \phi(t)$ in (5.2) is continuous and bounded on $R$. This shows that A is in $X$. So, $A$ maps from the Banach space $X$ into itself.

For $\phi, \psi$ in $X$ one readily sees that $\|A \phi-A \psi|| \beta\| \phi-\psi \|$. Since $\beta<1$, mapping $A$ is a strict contraction. This proves that there exists a unique continuous bounded solution $x(t)$ of $(1.1)$ on $R$.

Using the argument of Burton [15] we see that $x$ is T-periodic. Indeed, from Lemma 4 we know that $x(t+T)$ is also a continuous bounded solution of (1.1) on $R$. Since $x(t)$ is the only continuous bounded solution of (1.1) on $R$, it follows that $x(t)=x(t+T)$ for all $-\infty<t<\infty$. This completes the proof of Theorem 5 .

REMARK. From the proof of Theorem 5 it may appear that the existence and uniqueness of a continuous periodic solution $x(t)$ of (1.1) on $R$ could be obtained by defining the map $A$ from $P_{T}$ into itself instead of $X$ into itself where $P_{T}$ is the Banach space introduced at the beginning of this section. In that case the use of Lemma 4 could be avoided. However, this would not prove that the solution $x(t)$ is the only continuous bounded solution on $R$. 
THEOREM 6. Suppose (Al) - (A7) hold. Consider B of (A4). For any $\alpha>0$ with $\alpha \beta<1$ choose $\eta$ of (A7). Then for each $f$ satisfying $|f(t)|<(1-\alpha \beta) \eta$ as well as assumption (Al) there exists a unique continuous periodic solution $x(t)$ of (1.2) with $|x(t)|<n$ on $R$. Moreover, the function $x(t)$ is the only continuous solution of (1.2) with $|x(t)|<n$ on $R$.

PROOF. Fix $\alpha>0$ with $\alpha \beta<1$. Then from (A7) it follows that there exists an $n>0$ such that $|g(t, x)|<\alpha|x|$ uniformly in $-\infty<t<\infty$ whenever $|x|<n$. Choose $f$ satisfying ( $\mathrm{Al}$ ) and the condition $|f(t)|<(1-\alpha \beta)$ for all $-\infty<t<\infty$. Consider the set.

$$
S=\left\{\phi(t): R \rightarrow R^{n}, \phi(t) \text { is continuous, }|\phi(t)|<n \text { for all }-\infty<t<\infty\right\}
$$

For $\phi$ in $S$, define a map $A$ on $S$ by

$$
A \phi(t)=f(t)+\int_{-\infty}^{t} k(t, s) g(s, \phi(s)) d s .
$$

It follows from (A7) and Lemma 5 that $\int_{-\infty}^{t} k(t, s) g(s, \phi(s)) d s$ is continuous on $R$. Therefore, the function $A \phi(t)$ in (5.3) is continuous on $R$. Again, $|A \phi(t)|<(1-\alpha \beta) n+\alpha \beta n=n$. So, A maps from $S$ into itself. Finally, for $\phi, \psi$ in $S$, ||$A \phi-A \psi||<\alpha \beta|| \phi-\psi||$. Since $\alpha \beta<1$, mapping $A$ is a strict contraction. This proves that there exists a unique continuous solution $x(t)$ of (1.2) with $|x(t)|<n$ on $R$. It follows from Lemma 4 that the function $x(t+T)$ is also a continuous solution of (1.2) with $|x(t+T)|<\eta$ on $R$. This shows that $x(t)=x(t+T)$ for all $t$ in $R$ as required.

REMARK. Theorems 5 and 6 hold even if we replace assumption (A5) by (4.7). Condition (4.7) will provide the required continuity of $A \phi(t)$ in (5.2) and (5.3).

\section{ACKNOWLEDGMENTS}

This work is part of the author's Ph.D. dissertation written under the direction of Professor T.A. Burton. The author gratefully acknowledges Professor Burton's guidance throughout this work.

\section{REFERENCE $S$}

1. MILLER, R.K. Nonlinear Volterra Integral Equations, Benjamin, Menlo Park, California, 1971.

2. GRIPENBERG, G. On the resolvents of nonconvolution Volterra kernels, Funkcialaj Ekvacioj, 23 (1980), 83-95.

3. STRAUSS, A. On a perturbed volterra integral equation, J. Math. Anal. Appl., 30 (1970), 564-575.

4. PALEY, R.E.A.C. and WIENER, N. Fourier transforms in the complex domain, Amer. Math. Soc. Colloquium Publications, 1934.

5. GRIPENBERG, G. On positive nonincreasing resolvents of Volterra equations, J. Differential Equations, 30 (1978), 380-390. 
6. GRIPENBERG, G. On the resolvents of Volterra equations with nonincreasing kernels, J. Math. Ana1. App1., 76 (1980), 134-145.

7. GRIPENBERG, G. On the asymptotic behavior of resolvents of Volterra equations, SIAM J. Math. Ana1., 11 (1980), 654-662.

8. GROSSMAN, S.I. Integrability of resolvents of certain Volterra integral equations, J. Math. Ana1. App1., 48 (1974), 785-793.

9. MILLER, R.K. On Volterra integral equations with nonnegative integrable resolvents, J. Math. Anal. Appl., 22 (1968), 319-340.

10. LEITMAN, M.J. and MIZEL, V.J. Asymptotic stability and the periodic solutions of $x(t)+\int_{-\infty}^{t} a(t-s) g(s, x(s)) d s=f(t)$, J. Math. Anal. Appl., 66 (1978), 606-625.

11. ISLAM, M.N. Periodic solutions of nonlinear integral equations, Annali di Mat. Pura ed App1., to appear.

12. ELOE, P.W. and ISLAM, M.N. Periodic solutions of nonlinear integral equations with infinite memory, Applicable Analysis, to appear.

13. BURTON, T.A., Stability and Periodic Solutions of Ordinary and Functional Differential Equations, Mathematics in Science and Engineering, Vol. 178, Academic Press, New York, 1985.

14. BURTON, T.A. Periodicity and limiting equations in Volterra systems, Bo11. Un. Mat. Ital. Serie VI, Vol. IV-C, N.1 (1985), 31-39.

15. BURTON, T.A. Periodic solutions of linear Volterra equations, Funkcialaj Ekvacioj, 27 (1984), 229-253.

16. LANGENHOP, C.E. Periodic and almost periodic solutions of Volterra integral differential equations with infinite memory, J. Differential Equations, 58 (1985), 391-403.

17. ARINO, 0 . and HADDOCK, J. Estimates for periodic solutions of diferential equations with inifinite delay, Notices Amer. Math. Soc. (29) $\underline{5}$ (1984), 57.

18. BURTON, T.A. Volterra integral and differential equations, Mathematics in Science and Engineering, Vo1. 167, Academic Press, New York, 1983. 


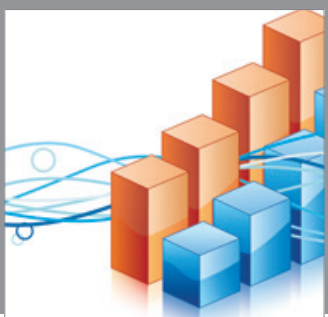

Advances in

Operations Research

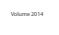

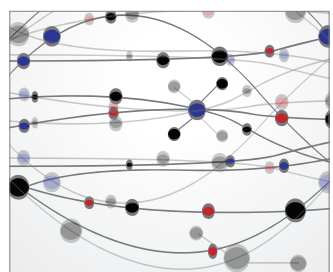

\section{The Scientific} World Journal
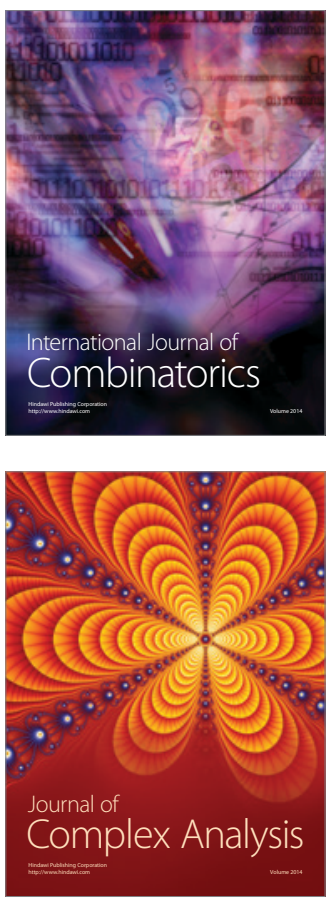

International Journal of

Mathematics and

Mathematical

Sciences
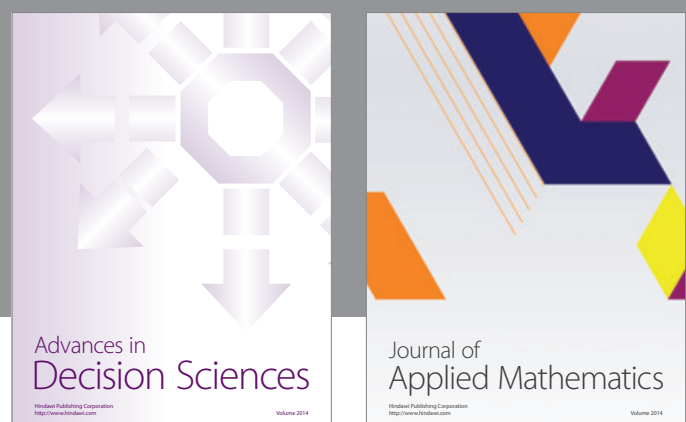

Journal of

Applied Mathematics
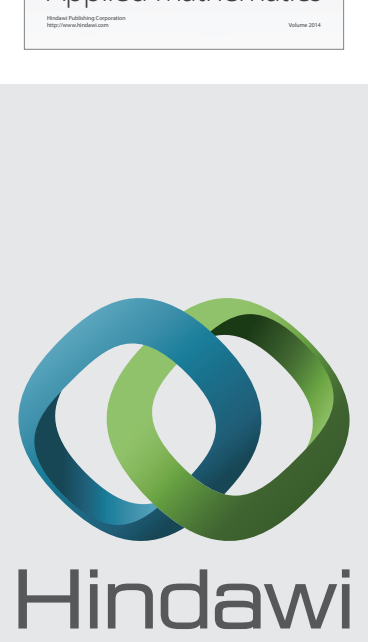

Submit your manuscripts at http://www.hindawi.com
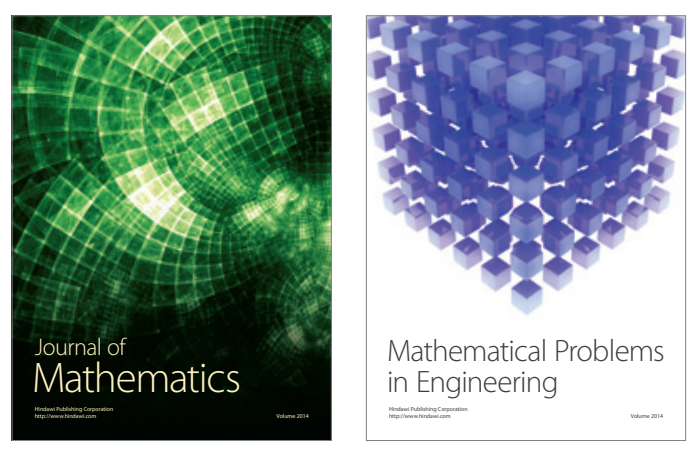

Mathematical Problems in Engineering
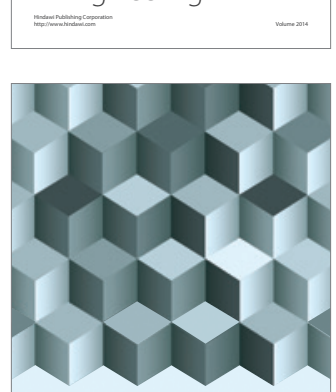

Journal of

Function Spaces
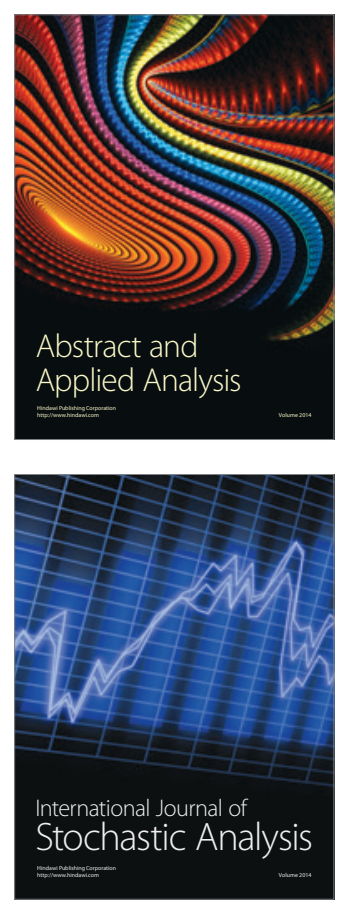

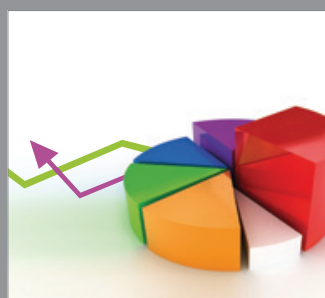

ournal of

Probability and Statistics

Promensencen
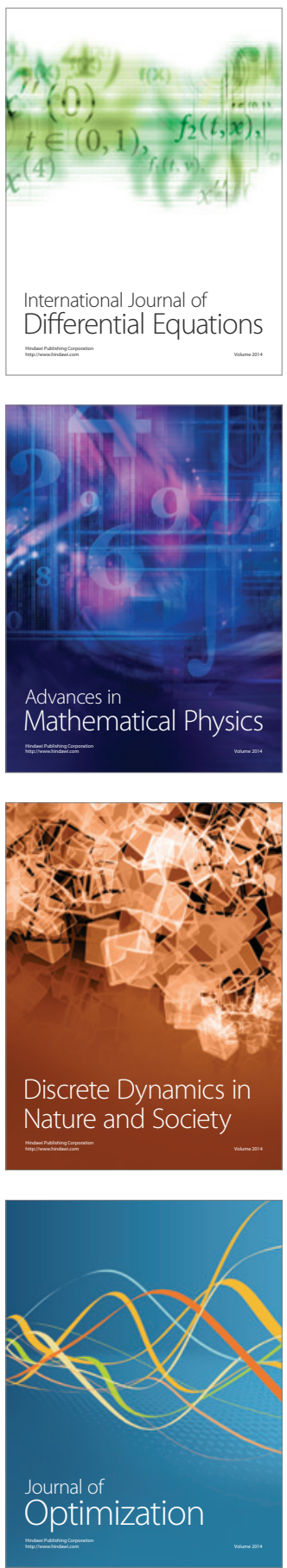